\title{
A DIVERGENT WEIGHTED ORTHONORMAL SERIES OF BROKEN LINE FRANKLIN FUNCTIONS
}

\author{
BY
}

COKE S. REED

\begin{abstract}
The purpose of this paper is to define a differentiable function $F$ and an inner product on the space of continuous functions on $[0,1]$ in such a way that the Fourier expansion of $F$ obtained by orthonormalizing the broken line Franklin functions according to this inner product is divergent.
\end{abstract}

I. Introduction. Let $z_{1}, z_{2}, \ldots$ denote a reversible number sequence such that $\left\{z_{i}\right\}$ is a subset of $[0,1]$ and is dense in $[0,1]$. Define $\theta_{0}, \theta_{1}, \theta_{2}, \ldots$ to be the sequence of functions on $[0,1]$ such that $\theta_{0}(x)=1$, and for each positive integer $i, \theta_{i}(x)=0$ if $0 \leq x \leq z_{i}$, and $\theta_{i}(x)=x-z_{i}$ if $z_{i} \leq x \leq 1$. Let $W$ denote a continuous strictly increasing function on $[0,1]$. Define the inner product $((f, g))_{W}$ of two continuous functions $f$ and $g$ with domain $[0,1]$ to be $\int_{0}^{1} f \cdot g d W$. Since no member of the sequence $\theta_{0}, \theta_{1}, \theta_{2}, \ldots$ is a finite linear combination of the other members of the sequence, we can use the Gram-Schmidt process to construct a sequence $\phi_{0}, \phi_{1}, \phi_{2}, \ldots$ of functions on $[0,1]$ such that for each positive integer $k, \phi_{k}$ is a linear combination of $\theta_{0}, \theta_{1}, \theta_{2}, \ldots, \theta_{k}$ and $\theta_{k}$ is a linear combination of $\phi_{0}, \phi_{1}, \ldots, \phi_{k}$ and such that $\phi_{0}, \phi_{1}, \phi_{2}, \ldots$ is orthonormal relative to $((,))_{W}$. The sequence $\phi_{0}, \phi_{1}, \ldots$ will be referred to as the $(z, W)$ sequence. Let $I$ denote the function such that $I(x)=x$.

Franklin [1] showed that if $f$ is a continuous function with domain [0,1], and $z_{1}, z_{2}, \ldots$ satisfies a certain property and $\phi_{0}, \phi_{1}, \ldots$ is the $(z, I)$ sequence, then the sequence of functions $s_{n}=\sum_{i=0}^{n}\left(\left(f, \phi_{i}\right)\right) \cdot \phi_{i}$ converges uniformly to $f$ as $n$ tends to infinity. Wall [3] used $(z, W)$ sequences for arbitrary $z$ and $W$ in the study of certain moment problems and raised the following question to his students. Is it true that if $F$ is a continuous function defined on $[0,1]$ and $z_{1}, z_{2}, \ldots$ is a reversible number sequence such that $\left\{z_{i}\right\}$ is a subset of $[0,1]$ and is dense in $[0,1]$ and $W$ is a continuous and strictly increasing function defined on $[0,1]$, then $\sum_{i=0}^{n}\left(\left(f, \phi_{i}\right)\right)_{W} \cdot \phi_{i}$ converges uniformly to $f$ as $n$ tends to infinity? Sox [2] showed that for every such sequence $z$, the $(z, I)$ Fourier expansion of a continuous function $f$ converges uniformly to $f$. It is the purpose of this paper to exhibit a $z$, $W$, and $F$ so that if $\phi_{0}, \phi_{1}, \phi_{2}, \ldots$ is the $(z, W)$ sequence, then $\sum_{i=0}^{n}\left(\left(F, \phi_{i}\right)\right) \phi_{i}$ is divergent.

Presented to the Society, November 25, 1972; received by the editors December 5, 1972 and, in revised form, March 29, 1973.

AMS (MOS) subject classifications (1970). Primary 42A56, 42A60.

Key words and phrases. Franklin functions, Fourier series, weighted orthonormal series, broken line functions. 
II. The example. There exists a $z$ sequence, a differentiable function $F$ defined on $[0,1]$ and a strictly increasing infinitely differentiable function $W$ defined on $[0,1]$ such that if $\phi_{0}, \phi_{1}, \phi_{2}, \ldots$ is the $(z, W)$ sequence, then the number sequence $\sum_{i=0}^{n}\left(\left(\phi_{i}, F\right)\right) \cdot \phi_{i}(1)$ is unbounded.

Let $\left(z_{1}, z_{2}, \ldots\right)$ denote the sequence $(0,1 / 2,1 / 4,3 / 4,1 / 8,3 / 8,5 / 8,7 / 8,1 / 16, \ldots)$; let $K$ denote the set $\{1 / 2,1 / 4,1 / 8, \ldots\}$ and let $h$ denote the function defined by $h(x)$ $=(1-x)^{2}$. Two sequences $\left(f_{1}, f_{2}, \ldots\right)$ and $\left(w_{1}, w_{2}, \ldots\right)$ of functions will now be defined inductively so that the desired functions $F$ and $W$ of the example can be defined by the formulas $F=\sum_{i=1}^{\infty} f_{i}$ and $W=\sum_{i=1}^{\infty} w_{i}$. In order to facilitate this construction, fourteen additional sequences, $\left(N_{1}, N_{2}, \ldots\right),\left(M_{1}, M_{2}, \ldots\right),\left(t_{1}, t_{2}\right.$, $\ldots),\left(g_{1}, g_{2}, \ldots\right),\left(R_{1}, R_{2}, \ldots\right),\left(\alpha_{1}, \alpha_{2}, \ldots\right),\left(\beta_{1}, \beta_{2}, \ldots\right),\left(\lambda_{1}, \lambda_{2}, \ldots\right),\left(V_{1}, V_{2}\right.$, $\ldots),\left(d_{1}, d_{2}, \ldots\right),\left(s_{1}, s_{2}, \ldots\right),\left(u_{1}, u_{2}, \ldots\right),\left(c_{1}, c_{2}, \ldots\right)$ and $\left(P_{1}, P_{2}, \ldots\right)$, will be defined inductively along with the sequences $\left(f_{1}, f_{2}, \ldots\right)$ and $\left(w_{1}, w_{2}, \ldots\right)$.

Set $M_{1}=1 / 2$ and set $N_{1}=1 / 2$. Let $t_{1}$ denote a member of $K$ such that $t_{1}<M_{1} / 4$ and such that the function $g_{1}$ defined by the following formulas lies below $h$.

$$
\begin{aligned}
g_{1}(x) & =0, & & \text { if } 0 \leq x \leq N_{1}, \\
& =4 x / M_{1}-4 N_{1} / M_{1}, & & \text { if } N_{1} \leq x \leq N_{1}+t_{1}, \\
& =-4 x / M_{1}+4\left(N_{1}+2 t_{1}\right) / M_{1}, & & \text { if } N_{1}+t_{1} \leq x \leq N_{1}+2 t_{1}, \\
& =0, & & \text { if } N_{1}+2 t_{1}<x \leq 1 .
\end{aligned}
$$

Notice that $g_{1}$ is continuous. Let $f_{1}$ denote a differentiable function such that if $x \in[0,1] ;$ then $0 \leq f_{1}(x) \leq h(x)$ and if $x \in[0,1]-\left(\left[N_{1}-t_{1} / 4, N_{1}+t_{1} / 4\right]\right.$ $\left.\cup\left[N_{1}+3 t_{1} / 4, N_{1}+5 t_{1} / 4\right] \cup\left[N_{1}+7 t_{1} / 4, N_{1}+9 t_{1} / 4\right]\right)$, then $f_{1}(x)=g_{1}(x)$. Let $R_{1}$ denote the class of all straight lines $\gamma$ with the property that $\gamma(1)<1$. Set $\alpha_{1}=N_{1}+t_{1} / 3$, set $\beta_{1}=N_{1}+2 t_{1} / 3$ and set $\lambda_{1}=N_{1}+3 t_{1}$. Let $V_{1}$ denote the number set to which the number $d$ belongs if and only if there exists a member $\gamma$ of $R_{1}$ with the property that $d=\int_{\alpha_{1}}^{\beta_{1}}\left[\gamma(x)-f_{1}(x)\right]^{2} d x$. Let $\delta$ denote a straight line such that $1<\delta(1)<2$ and such that $\delta$ intersects $f_{1}$ at a point $(a, b)$ where $\alpha_{1}<a<\beta_{1}$. If $\gamma$ is in $R_{1}$, then either $\int_{\alpha_{1}}^{a}[\gamma(x)-\delta(x)]^{2} d x<\int_{\alpha_{1}}^{a}\left[\gamma(x)-f_{1}(x)\right]^{2} d x$ or else $\int_{a}^{\beta_{1}}[\gamma(x)-\delta(x)]^{2} d x<\int_{a}^{\beta_{1}}\left[\gamma(x)-f_{1}(x)\right]^{2} d x$. Therefore, the greatest lower bound of $V_{1}$ is positive. Let $d_{1}$ denote a positive number less than one and less than the greatest lower bound of $V_{1}$. Set $s_{1}=1$. Let $u_{1}$ denote a function defined on $[0,1]$ with the following seven properties:

(1) $u_{1}$ is infinitely differentiable over $[0,1]$.

(2) $u_{1}(0)=0$.

(3) $u_{1}$ is strictly increasing over $\left[0, \lambda_{1}\right]$.

(4) If $x$ and $y$ are in $\left[\lambda_{1}, 1\right]$, then $u_{1}(x)=u_{1}(y)$.

(5) The restriction of $u_{1}$ to $\left[\alpha_{1}, \beta_{1}\right]$ is a straight line with slope $s_{1}$.

(6) If $x \in[0,1]$, then $u_{1}^{\prime}(x) \leq s_{1}$.

(7) $\left[u_{1}\left(\alpha_{1}\right)-u_{1}(0)\right]+\left[u_{1}(1)-u_{1}\left(\beta_{1}\right)\right]<s_{1} \cdot d_{1} / 4$. 
There is a positive number $c_{1}<1$ such that for each number $x$ in $[0,1]$, $\left|c_{1} \cdot u_{1}^{\prime}(x)\right|<1 / 2$. Let $w_{1}=c_{1} \cdot u_{1}$.

Continue this construction inductively for each integer $j>1$ as follows. Let $M_{j}$ denote a member of $K$ satisfying the inequalities $\lambda_{j-1}<1-3 M_{j}$ and $M_{j}<t_{j-1}$. Let $N_{j}=1-M_{j}$. There is an integer $k$ such that $z_{k}=N_{j}$. There exists a $k+1$ term number sequence $a_{0}, a_{1}, \ldots, a_{k}$ such that if $b_{0}, b_{1}, \ldots, b_{k}$ is a $k+1$ term number sequence, then

$$
\begin{aligned}
& \int_{0}^{\lambda_{j-1}}\left(\sum_{n=1}^{j-1} f_{n}-\sum_{n=0}^{k} a_{n} \phi_{n}\right)^{2} d \sum_{n=1}^{j-1} w_{n} \\
& \quad \leq \int_{0}^{\lambda_{j-1}}\left(\sum_{n=1}^{j-1} f_{n}-\sum_{n=0}^{k} b_{n} \phi_{n}\right)^{2} d \sum_{n=1}^{j-1} w_{n} .
\end{aligned}
$$

Let $P_{j-1}=\sum_{n=0}^{k} a_{n} \phi_{n}$. Let $t_{j}$ denote a member of $K$ such that $t_{j}<M_{j} / 4$ and such that the function $g_{j}$, defined by the following formulas, lies below $h$.

$$
\begin{aligned}
g_{j}(x) & =0, & & \text { if } 0 \leq x \leq N_{j}, \\
& =4 j x / M_{j}-4 j N_{j} / M_{j}, & & \text { if } N_{j} \leq x \leq N_{j}+t_{j}, \\
& =-4 j x / M_{j}+4 j\left(N_{j}+2 t_{j}\right) / M_{j}, & & \text { if } N_{j}+t_{j} \leq x \leq N_{j}+2 t_{j}, \\
& =0, & & \text { if } N_{j}+2 t_{j}<x \leq 1 .
\end{aligned}
$$

Notice that $g_{j}$ is continuous. Let $f_{j}$ denote a differentiable function such that if $x \in[0,1]$, then $0 \leq f_{j}(x) \leq h(x)$ and if $x \in[0,1]-\left\{\left[N_{j}-t_{j} / 4, N_{j}+t_{j} / 4\right] \cup\left[N_{j}\right.\right.$ $\left.\left.+3 t_{j} / 4, N_{j}+5 t_{j} / 4\right] \cup\left[N_{j}+7 t_{j} / 4, N_{j}+9 t_{j} / 4\right]\right\}$, then $f_{j}(x)=g_{j}(x)$. Let $R_{j}$ denote the class of all straight lines $\gamma$ with the property that $\gamma(1)<j$. Set $\alpha_{j}=N_{j}+t_{j} / 3$, set $\beta_{j}=N_{j}+2 t_{j} / 3$, and set $\lambda_{j}=N_{j}+3 t_{j}$. Let $V_{j}$ denote the number set to which the number $d$ belongs if and only if there exists a member $\gamma$ of $R_{j}$ with the property that $d=\int_{\beta_{j}}^{\alpha_{j}}\left(\gamma(x)-f_{j}(x)\right)^{2} d x$. As with $V_{1}$, the greatest lower bound of $V_{j}$ is positive. Let $d_{j}$ denote a positive number less than $d_{j-1}$ and less than the greatest lower bound of $V_{j}$. Set $s_{j}=c_{j-1} \cdot s_{j-1} \cdot d_{j-1} /\left(64 j^{2}\right)$.

Let $u_{j}$ denote a function defined on $[0,1]$ with the following eight properties:

(1) $u_{j}$ is infinitely differentiable over $[0,1]$.

(2) If $x \in\left[0, \lambda_{j-1}\right]$, then $u_{j}(x)=0$.

(3) $u_{j}$ is strictly increasing on $\left[\lambda_{j-1}, \lambda_{j}\right]$.

(4) If $x$ and $y$ are in $\left[\lambda_{j}, 1\right]$, then $u_{j}(x)=u_{j}(y)$.

(5) The restriction of $u_{j}$ to $\left[\alpha_{j}, \beta_{j}\right]$ is a straight line with slope $s_{j}$.

(6) If $x \in[0,1]$, then $u_{j}^{\prime}(x) \leq s_{j}$.

(7) $\left[u_{j}\left(\alpha_{j}\right)-u_{j}(0)\right]+\left[u_{j}(1)-u_{j}\left(\beta_{j}\right)\right]<s_{j} \cdot d_{j} /\left(64 j^{2}\right)$.

(8) $u_{j}\left(\lambda_{j-1}+M_{j}\right)<s_{j} \cdot d_{j} /\left(32 j^{2}\left(\left|P_{j-1}\left(\lambda_{j-1}\right)\right|+1\right)^{2}\right)$.

There is a positive number $c_{j}<1$ such that for each $x$ in $[0,1]$ and each positive integer $k \leq j,\left|c_{j} \cdot u_{j}^{(k)}(x)\right|<1 / 2^{j}$ where $u_{j}^{(k)}$ is the $k$ th derivative of $u_{j}$. Let $w_{j}=c_{j} \cdot u_{j}$. 
Notice that if $x$ is a number in $[0,1)$ and there exists a positive integer $i$ such that $f_{i}(x) \neq 0$, then there is an open set $S$ containing $x$ such that if $j$ is a positive integer distinct from $i$ and $\psi$ is in $S$, then $f_{j}(\psi)=0$. It follows from this fact that the function $F=\sum_{i=1}^{\infty} f_{i}$ exists and is differentiable at each number $x$ in $[0,1)$. But for each $x \in[0,1]$, we have that $0 \leq F(x) \leq h(x)$ and therefore $F$ is differentiable at 1 .

If $m$ is a positive integer, and $j$ is an integer greater than $m$, and $x$ is a number in $\left[1-2 M_{j}, 1\right]$, then $\left|W^{(m)}(x)\right|<1 / 2^{j}$. Notice that this fact implies that $W$ and all of its derivatives exist on $[0,1]$ and $W^{(m)}(1)=0$.

Suppose that there exists an integer $j>1$ such that if $k$ is the integer with the property that $z_{k}=N_{j}$, then $\sum_{i=0}^{k}\left(\left(\phi_{i}, F\right)\right)_{W} \phi_{i}(1)<j$. Let $G=\sum_{i=0}^{k}\left(\left(\phi_{i}, F\right)\right)_{W} \phi_{i}$. $G$ has the property that if $a_{0}, a_{1}, \ldots, a_{k}$ is a $k+1$ term number sequence, then

$$
\int_{0}^{1}(F-G)^{2} d W \leq \int_{0}^{1}\left(F-\sum_{i=0}^{k} a_{i} \phi_{i}\right)^{2} d W .
$$

Since $\lambda_{j-1}<N_{j}<\alpha_{j}<\beta_{j}$, we have that

$$
\int_{0}^{1}(F-G)^{2} d W \geq \int_{0}^{\lambda_{j-1}}(F-G)^{2} d W+\int_{\alpha_{j}}^{\beta_{j}}(F-G)^{2} d W .
$$

But the restriction of $F$ to $\left[\alpha_{j}, \beta_{j}\right]$ is a subset of $f_{j}$ and the restriction of $G$ to $\left[\alpha_{j}, \beta_{j}\right]$ lies on a straight line $\gamma$ such that $\gamma(1)<j$. Therefore, we have that $\int_{\alpha_{j}}^{\beta_{j}}(F-G)^{2} d W=\int_{\alpha_{j}}^{\beta_{j}}\left(f_{j}-\gamma\right)^{2} d W$. But the restriction of $W$ to $\left[\alpha_{j}, \beta_{j}\right]$ lies on a straight line with slope $c_{j} \cdot s_{j}$ and therefore

$$
\int_{\beta_{j}}^{\alpha_{j}}\left(f_{j}-\gamma\right)^{2} d W=c_{j} \cdot s_{j} \cdot \int_{\alpha_{j}}^{\beta_{j}}\left(f_{j}-\gamma\right)^{2}(x) d x .
$$

Since $\gamma$ is in $R_{j}, \int_{\beta_{j}}^{\alpha_{j}}\left(f_{j}-\gamma\right)^{2}(x) d x>d_{j}$. Combining these inequalities gives the inequality

$$
\int_{0}^{1}(F-G)^{2} d W>\int_{0}^{\lambda_{j-1}}(F-G)^{2} d W+c_{j} \cdot s_{j} \cdot d_{j} .
$$

Let $H$ denote the function with the following four properties:

(1) The restriction of $H$ to $\left[0, \lambda_{j-1}\right]$ is $P_{j-1}$.

(2) The restriction of $H$ to $\left[\lambda_{j-1}, \lambda_{j-1}+M_{j}\right]$ lies on the straight line containing $\left(\lambda_{j-1}, P_{j-1}\left(\lambda_{j-1}\right)\right)$ and $\left(\lambda_{j-1}+M_{j}, 0\right)$.

(3) If $x \in\left[\lambda_{j-1}+M_{j}, N_{j}\right]$, then $H(x)=0$.

(4) The restriction of $H$ to $\left[N_{j}, 1\right]$ is a straight line such that if $x \in\left[\alpha_{j}, \beta_{j}\right]$, then $H(x)=F(x)$.

There is a $k+1$ term number sequence $a_{0}, a_{1}, \ldots, a_{k}$ such that $H(x)$ $=a_{0} \phi_{0}+a_{1} \phi_{1}+\cdots+a_{k} \phi_{k}$, and therefore,

$$
\begin{aligned}
\int_{0}^{1}(F-H)^{2} d W & \geq \int_{0}^{1}(F-G)^{2} d W \\
& >\int_{0}^{\lambda_{j-1}}(F-G)^{2} d W+c_{j} \cdot s_{j} \cdot d_{j} .
\end{aligned}
$$


Notice that

$$
\begin{aligned}
\int_{0}^{1}(F-H)^{2} d W= & \int_{0}^{\lambda_{j-1}}(F-H)^{2} d W+\int_{\lambda_{j-1}}^{\lambda_{f-1}+M_{j}}(F-H)^{2} d W \\
& +\int_{\lambda_{j-1}+M_{j}}^{\alpha_{j}}(F-H)^{2} d W+\int_{a_{j}}^{\beta_{j}}(F-H)^{2} d W \\
& +\int_{\beta_{j}}^{1}(F-H)^{2} d W .
\end{aligned}
$$

For each $x \in\left[0, \lambda_{j-1}\right], F(x)=\sum_{i=1}^{j-1} f_{i}(x)$ and $H(x)=P_{j-1}(x)$. Therefore, from the definition of $P$, we obtain:

$$
\begin{aligned}
\int_{0}^{\lambda_{-1}}(F-H)^{2} d W & =\int_{0}^{\lambda_{-1}}\left(\left(\sum_{i=1}^{j-1} f_{i}\right)-P_{j-1}\right)^{2} d W \\
& \leq \int_{0}^{\lambda_{j-1}}(F-G)^{2} d W .
\end{aligned}
$$

For each $x \in\left[\lambda_{j-1}, \lambda_{j-1}+M_{j}\right], F(x)=0$ and $|H(x)| \leq\left|P_{j-1}\left(\lambda_{j-1}\right)\right|$. Moreover,

$$
W\left(\lambda_{i j}+M_{j}\right)-W\left(\lambda_{j-1}\right)=w_{j}\left(\lambda_{j-1}+M_{j}\right)-w_{j}\left(\lambda_{j-1}\right)<\frac{c_{j} \cdot s_{j} \cdot d_{j}}{4 j\left(\left(P_{j-1}\left(\lambda_{j-1}\right) \mid+1\right)^{2}\right.}
$$

Therefore, we have that

$$
\begin{aligned}
\int_{\lambda_{j-1}}^{\lambda_{j-1}+M_{j}}(F-H)^{2} d W & \leq \frac{\left(P_{j-1}\left(\lambda_{j-1}\right)\right)^{2} \cdot c_{j} \cdot s_{j} \cdot d_{j}}{\left(4 j\left(P_{j-1}\left(\lambda_{j-1}\right)+1\right)^{2}\right)} \\
& <\frac{c_{j} \cdot s_{j} \cdot d_{j}}{4} .
\end{aligned}
$$

For each $x \in\left[\lambda_{j-1}+M_{j}, \alpha_{j}\right],(F-H)^{2}(x)<1$. Moreover, $W\left(\alpha_{j}\right)-W\left(\lambda_{j-1}\right.$ $\left.+M_{j}\right)=w_{j}\left(\alpha_{j}\right)-w_{j}\left(\lambda_{j-1}+M_{j}\right)<c_{j} \cdot s_{j} \cdot d_{j} / 4$. There inequalities imply

$$
\int_{\lambda_{j-1}+M_{j}}^{a_{j}}(F-H)^{2} d W<c_{j} \cdot s_{j} \cdot d_{j} / 4 \text {. }
$$

For each $x \in\left[\alpha_{j}, \beta_{j}\right], F(x)=H(x)$ and therefore,

$$
\int_{\alpha_{j}}^{\beta_{j}}(F-H)^{2} d W=0 .
$$

For each $x \in\left[\beta_{j}, 1\right],(F-H)^{2}(x)<16 j^{2}$. Moreover,

$$
\begin{aligned}
W(1)-W & \left(\beta_{j}\right)=w_{j}(1)-w_{j}\left(\beta_{j}\right)+w_{j+1}(1)-w_{j+1}\left(\beta_{j}\right)+w_{j+2}(1)-w_{j+2}\left(\beta_{j}\right)+\cdots \\
& <c_{j} s_{j} d_{j} /\left(64 j^{2}\right)+c_{j+1} s_{j+1} \cdot\left(\lambda_{j+1}-\lambda_{j}\right)+c_{j+2} s_{j+2} \cdot\left(\lambda_{j+2}-\lambda_{j+1}\right)+\cdots \\
& <c_{j} s_{j} d_{j} /\left(32 j^{2}\right) .
\end{aligned}
$$

Therefore, we have the inequality

$$
\int_{\beta_{j}}^{1}(F-H)^{2} d W<\left(16 j^{2}\right)\left(s_{j} d_{j} c_{j} /\left(32 j^{2}\right)\right)<c_{j} s_{j} d_{j} / 2 .
$$


Combining inequalities (B), (C), (D), (E), (F), and (G) yields

$$
\int_{0}^{1}(F-H)^{2} d W<\int_{0}^{\lambda_{j-1}}(F-G)^{2} d W+c_{j} s_{j} d_{j}
$$

which contradicts inequality (A) and therefore the proof is completed.

This example gives rise to a number of questions:

(1) Characterize those strictly increasing continuous functions $w$ which have the property that if $f$ is a continuous function on 0,1 and $z_{1}, z_{2}, \ldots$ is a $z$ sequence, then the $(z, w)$ Fourier expansion of $f$ converges uniformly to $f$.

(2) By a slight modification of the example in this paper, one can construct a $z, f$, and $w$ such that the $(z, w)$ Fourier expansion of $f$ converges pointwise to $f$ but not uniformly to $f$. However, the following question remains unanswered: Is there a $(z, w)$ system such that if $f$ is a continuous function on $[0,1]$ then the $(z, w)$ Fourier expansion of $f$ converges pointwise to $f$, but there exists a continuous $g$ on $[0,1]$ such that the $(z, w)$ Fourier expansion of $g$ does not converge uniformly to $g$ ?

(3) Is there a strictly increasing continuous $w$ such that there exist sequences $z$ and $z^{\prime}$ such that if $f$ is continuous on $[0,1]$ then the $(z, w)$ Fourier expansion of $f$ converges uniformly to $f$, but there exists a continuous function $g$ on $[0,1]$ such that the $\left(z^{\prime}, w\right)$ Fourier expansion of $g$ does not converge to $g$ ?

(4) Is the following statement a theorem: If $w$ is a strictly increasing continuously differentiable function on $[0,1]$ and $z_{1}, z_{2}, \ldots$ is a $z$ sequence in $[0,1]$ and $f$ is continuously differentiable on $[0,1]$, then the $(z, w)$ Fourier expansion of $f$ converges uniformly to $f$ over $[0,1]$.

\section{REFERENCES}

1. Philip Franklin, A set of continuous orthogonal functions, Math. Ann. 100 (1928), 522-529.

2. J. L. Sox, Jr., A class of complete orthogonal sequences of broken line functions, Trans. Amer. Math. Soc. 166 (1972), 293-296. MR 45 \#811.

3. H. S. Wall, Creative mathematics, Univ. of Texas Press, Austin, Tex., 1963, pp. 161-168.

Department of Mathematics, Auburn University, Auburn, Alabama 36830 